\section{A3.13 INVESTIGATING T-CELL SUBSETS IN LYMPH NODE BIOPSIES OF AUTOANTIBODY POSITIVE INDIVIDUALS AND EARLY ARTHRITIS PATIENTS}

doi:10.1136/annrheumdis-2013-203216.13

1,2TH Ramwadhdoebe, ' $\mathrm{J}$ Hähnlein, ${ }^{2} \mathrm{KI}$ Maijer, J Boorsma, ${ }^{3} \mathrm{LJ}$ van Boven, 'DM Gerlag, 'PP Tak, ${ }^{1,2, *}$ LGM van Baarsen. 'Division of Clinical Immunology and Rheumatology, Academic Medical Center, University of Amsterdam, Amsterdam, The Netherlands; ${ }^{2}$ Department of Experimental Immunology, Academic Medical Center, University of Amsterdam, Amsterdam, The Netherlands; ' ${ }^{3}$ Department of Radiology, Kennermergasthuis, Haarlem, The Netherlands

Background Rheumatoid arthritis (RA) is an immune-mediated inflammatory disease of unknown aetiology. Recent work has shown that systemic autoimmunity precedes inflammation of the synovium in RA patients. We developed a method to study the cellular composition of lymph nodes in the earliest phases of RA.

Objective Cross-sectional analysis of the phenotype and functional characteristics of $T$ cells from lymph nodes of individuals in different phases of rheumatoid arthritis.

Materials and Methods Seven individuals with arthralgia but without any evidence of arthritis upon physical examination who were/but positive for IgM rheumatoid factor (IgM-RF) and/or anti-citrullinated protein antibodies (ACPA; RA risk group) and 7 DMARD and biological naïve RA patients were included in the study. All study subjects underwent ultrasound-guided inguinal lymph node biopsy. T cell subsets T-helper (Th)1, cytotoxic T cell (Tc)1, Th2, Tc2, Th17, Tc17, regulatory T cells (Treg) and follicular $\mathrm{T}$ cells (Tfh) were analysed by multi-colour flow cytometry. Cytokine profiles were determined after stimulation with Phorbol Myristate Acetate (PMA) and Ionomycin in the presence of Brefeldin A and Golgi Stop. We used directly labelled antibodies for CD45, CD3, CD4, CD8, IFN-y (Th1/Tc1), IL-4 (Th2/Tc2), IL-17A (Th17/Tc17) Foxp3, and IL-10 (Treg).

Results Different T-helper cell subsets could be distinguished in the RA risk and arthritis group. An increase of CD4+IL-17A T cells (Th17; $p=0.04)$ and of CD4+IL-10 producing T cells $(p=0.014)$ could be observed in the early arthritis group compared to the RA risk group. Interestingly, a significant correlation between CD4+IL-10 producing T cells and ACPA titers $(r=0.78 ; p=0.0016)$ and between CD4+IL-17A producing $\mathrm{T}$ cells and IgM-RF levels $(r=0.57 ; p=0.04)$ was found when combining both study groups. In addition, within the RA risk group the levels of CD4+ IL-10 producing $\mathrm{T}$ cells correlated significantly with the TOTTJC28 $(r=0.8097 ; p=0.03)$. Within the early arthritis group no significant correlations between $\mathrm{T}$ cell subsets and disease activity were found.

Conclusions Flow cytometry analysis of cells collected using ultrasound-guided inguinal lymph node biopsy suggests an increase in activated T cells producing IL-17A and IL-10 in patients with early RA. Moreover, the number of these $\mathrm{T}$ cells correlates with autoantibody levels suggesting an important role of these $T$ cells in the humoral autoimmune response. These data indicate that inflammatory changes in lymph nodes are present during the earliest phases of RA which may provide new insights in early immunological changes associated with RA pathogenesis.

\section{A3.14 PD-1 SIGNALLING AND LOW RORC MRNA CONTENT INFLUENCE THE TREG/TH17 BALANCE IN HUMAN CORD BLOOD}

doi:10.1136/annrheumdis-2013-203216.14

'Sytze de Roock, 'Sanne Hoeks, 'Arie Jan Stoppelenburg, 'Berent Prakken, 2 Ismé de Kleer, 'Marianne Boes. ${ }^{1}$ Center for Molecular and Cellular Intervention CMCl, University Medical Center Utrecht, Utrecht, The Netherlands; ' Laboratory of Immunoregulation and Mucosal Immunology, Ghent University, Ghent, Belgium
Background and Objectives In neonates, the immune system is less responsive than in adults. Explanations for this involve differences in Thelper (Th) 1 versus Th2 cell numbers and altered cytokine profiles after stimulation. We hypothesised that neonatal immune deviation may also involve $\mathrm{FOXP3}^{+}$regulatory $\mathrm{T}$ cells (Treg) and Th17 cells

Materials and Methods In order to investigate this, we compared $\mathrm{CD}^{+}{ }^{+} \mathrm{CD} 25^{-} \mathrm{CD} 45 \mathrm{RO}^{-}$naïve $\mathrm{T}$ cells from human cord blood (CB) with cells from adult peripheral blood (APB). Cells were activated in vitro by anti-CD3 in the presence of viable antigen presenting cells (APB). The induction of regulatory $\mathrm{T}$ cells and Th17 cells was analysed by flow cytometry. Cytokine production was measured by multiplex immunoassays and RORC mRNA content by PCR. Specific interactions between APC and T cells were blocked with monoclonal antibodies during cell culture.

Results CB cells show lower numbers of Treg ex vivo than APB. However, upon activation, high percentages of functionally suppressive $\mathrm{FOXP3}^{+}$Treg are induced in $\mathrm{CB}$. Replacement of viable APC by irradiated APC or anti-CD28 abrogated this effect, suggesting that live APC from CB in particular may imprint a Treg phenotype. Indeed, $\mathrm{CB}$ APC were able to induce high numbers of $\mathrm{FOXP}^{+}$cells in alloreactive immune reactions with APB T cells as well. Addition of blocking antibodies against CD80, CD86 or CTLA-4 to CB cultures did not affect the number of Tregs induced. Blockade of the PD-1/PD-Ligand (PD-L)1 interaction however showed a marked decrease in $\mathrm{FOXP}^{+}$cell numbers. $\mathrm{CB}$ cells showed a consistently reduced protein kinase $\mathrm{B}(\mathrm{PKB}) / \mathrm{c}-\mathrm{Akt}$ phosphorylation upon activation. On top of that, in contrast to APB, low concentrations of APC derived pro-inflammatory cytokines were detected in $\mathrm{CB}$ cultures and no Th17 cells were induced. Addition of Th17 inducing cytokines reduced $\mathrm{FOXP3}^{+}$cell numbers in $\mathrm{CB}$, but did not induce IL-17 production. PCR of the Th17 defining transcription factor RORC showed a significantly reduced concentration of this protein in $\mathrm{CB}$. Conclusions Human cord blood cells have an increased propensity to become FOXP3 ${ }^{+}$Treg as compared to APB. This effect is due to low production of inflammatory cytokines and PD-1/PD-L1 interaction. Inability to induce Th17 cells in CB could not be abrogated by Th17 inducing cytokines, but was due to reduced RORC mRNA content. This study shows mechanisms involving the human Th17/ Treg induction switch that can also be important in inflammation and auto-immune disorders.

\section{A3.15 RECONSTITUTED HIGH DENSITY LIPOPROTEIN (rHDL) MODULATES T EFFECTOR CELL FUNCTION IN A MURINE MODEL OF RHEUMATOID ARTHRITIS}

doi:10.1136/annrheumdis-2013-203216.15

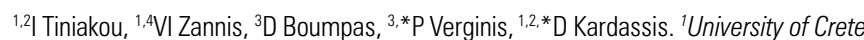
Medical School, Heraklion, Greece; ${ }^{2}$ Institute of Molecular Biology and Biotechnology, Foundation for Research and Technology of Hellas, Heraklion, Greece; 'Biomedical Research Foundation of the Academy of Athens, Greece; ${ }^{4}$ Boston University Medical Center, Boston, USA

\section{${ }^{*}$ Equal contribution}

Background and Objectives High density lipoprotein (HDL) has a variety of functions which confer protection from cardiovascular and other human diseases. Increased cardiovascular risk is observed in humans with autoimmune disorders such as rheumatoid arthritis (RA), which is associated with either low levels or dysfunctional HDL. Recent evidence suggests a role of HDL in modulating both innate and adaptive immune responses. We sought to investigate the role of $\mathrm{rHDL}$ during the development of an autoimmune response in the antigen-induced arthritis (AIA) mouse model.

Materials and Methods C57BL/6 mice were subcutaneously immunised with ovalbumin (OVA) in complete Freund's adjuvant (CFA) and the inguinal lymph nodes (LN) were excised 9 days after the antigenic challenge. The LN cells were cultured in vitro in the 
presence of varying concentrations of reconstituted $\mathrm{HDL}$ ( $\mathrm{rHDL}$ ) in the presence or absence of OVA and OVA-specific immune responses were measured. To assess the effect of $\mathrm{HDL}$ on dendritic cell activation and maturation, mouse bone marrow was cultured with GM-CSF to generate dendritic cells (BM-DCs), which were collected, cultured and treated with LPS in the presence or absence of rHDL.

Results OVA-primed LN cells secreted increased levels of IFN- $\boldsymbol{\gamma}$ and IL-17 that were significantly suppressed in the presence of rHDL in a dose-dependent manner. rHDL was also found to exert a suppressive effect on T cell proliferation as indicated by IL-2 measurement. Finally, rHDL-treated LPS-stimulated BM-DCs did not demonstrate any significant phenotypic differences as assessed by FACS analysis on CD86, CD40 and PDL-1 molecules as compared to control LPS-stimulated BM-DCs.

Conclusions rHDL exerts a direct immunomodulatory function on T cells in vitro by suppressing their proliferation and the expression of inflammatory cytokines. Ongoing work is focused on the delineation of the mechanism involved in the rHDL-mediated suppression of the immune response both in vitro and in vivo. These data identify rHDL as an important player in the homeostatic regulation of the inflammatory response and a potential therapeutic target for chronic inflammatory diseases.

\section{A3.16 SPECIFIC OVEREXPRESSION OF SYNOVIAL INTERLEUKIN-21 + CD4 ${ }^{+}$T CELLS CO-EXPRESSING TUMOUR NECROSIS FACTOR IN RHEUMATOID ARTHRITIS: ROLE IN JOINT DESTRUCTION?}

doi:10.1136/annrheumdis-2013-203216.16

${ }^{1,2} \mathrm{M}$ Cristina Lebre, ${ }^{3}$ Pedro L Vieira, 1,2Saida Aarrass, ${ }^{3}$ Thomas Newsom-Davis, 'Paul P Tak, ${ }^{3}$ Gavin R Screaton. 'Division of Clinical Immunology and Rheumatology; ${ }^{2}$ Department Experimental Immunology, Academic Medical Center/University of Amsterdam, Amsterdam, The Netherlands; ${ }^{3}$ Department of Immunology, Imperial College London, Hammersmith Campus, London, UK

Background and Objectives IL-21 is a cytokine produced by activated $\mathrm{CD}^{+} \mathrm{T}$ cells and $\mathrm{T}$ follicular helper cells (TFh) that has been implicated in several autoimmune diseases. IL-21 regulates antibody production by $\mathrm{B}$ cells and induces osteoclastogenesis, mechanisms that contribute to rheumatoid arthritis (RA) pathology. Importantly, IL-21R blockade ameliorates arthritis in mice. Here we investigated the functional characteristics of CD4+IL-21 + T cells in RA.

Materials and Methods We evaluated the expression of surface markers and cytokine production in matched peripheral blood (PB) and synovial fluid (SF) from $13 \mathrm{RA}$ and 6 psoriatic arthritis (PsA) patients, and $\mathrm{PB}$ of 17 healthy control (HC) subjects by flow cytometry following PMA/Ionomycin stimulation ex-vivo. IL-21 concentrations were assessed by ELISA in cell-free SF samples of RA $(\mathrm{n}=15), \operatorname{Ps}(\mathrm{n}=14)$ and $\mathrm{OA}(\mathrm{n}=6)$ patients and in synovial biopsy culture supernatants ( 6 days) of RA $(n=6)$ and ankylosing spondylitis (AS; $n=5)$ patients. The effects of IL-21 were evaluated on cytokine and matrix metalloproteinase (MMP) release by RA synovial biopsies.

Results The frequency of IL-21+CD4 + T cells in RA and PsA SF was significantly higher compared to $\mathrm{PB}(\mathrm{P}<0.0001$ and $\mathrm{P}<0.0001$, respectively). Moreover, the frequency of both total IL-21+CD4+ $(\mathrm{P}=0.0140)$ and IL-21+TNF+CD4 + T cells $(\mathrm{P}=0.0038)$ in RA SF were significantly increased compared to PsA patients. The frequency of IL-21 + CD4 + T cells in RA PB was positively correlated with DAS28 ( $r=0.592, P=0.033)$, serum anti-cyclic citrullinated peptide (anti-CCP) antibodies $(\mathrm{r}=0.788, \mathrm{P}=0.001)$ and IgM-rheumatoid factor (IgM-RF; $r=0.691, \mathrm{P}=0.009$ ). In addition, the percentage of IL-21 + CD4 + T cells in anti-CCP + or IgM-RF + patients was significantly higher compared to anti-CCP- $(\mathrm{P}=0.03)$ and
IgM-RF- $(\mathrm{P}=0.01)$ patients respectively. In addition to $\mathrm{PB}$ and $\mathrm{SF}$, we found IL-21+CD4+ T cells present in RA synovial tissue. Significant higher levels of IL-21 in RA SF compared to OA SF $(\mathrm{P}=0.04)$ were observed confirming previous observations. RA synovial biopsies released significantly higher levels of IL-21 compared to biopsies from AS patients. The levels of IL-21 in SF were associated with CRP and with the MMP-1 and MMP-3. Related to this, IL-21 selectively induced MMP-1 and MMP-3 secretion by RA synovial biopsies. Synovial IL-21+CD4+ T cells did not phenotypically fit the TFh cell paradigm in that they did not express CXCR5 and ICOS.

Conclusions The results of this study support the notion that IL21-producing CD4 $\mathrm{T}$ cells are involved in promoting synovial inflammation (TNF) and joint destruction (MMP) in RA and might be a therapeutic target in this disease.

\section{A3.17 SYNOVIAL FIBROBLASTS DIRECTLY INDUCE TH17 PATHOGENICITY VIA THE CYCLOOXYGENASE/ PROSTAGLANDIN-E, PATHWAY, INDEPENDENT OF IL-23}

doi:10.1136/annrheumdis-2013-203216.17

1,2Sandra MJ Paulissen, 1,2Jan Piet van Hamburg, 1,2Nadine Davelaar, 1,2Patrick $S$ Asmawidjaja, ${ }^{1}, 2$ Johanna MW Hazes. ${ }^{1}$ Erik Lubberts. 'Department of Rheumatology, Erasmus MC, University Medical Center Rotterdam, The Netherlands; '2Department of Immunology, Erasmus MC, University Medical Center Rotterdam, The Netherlands

Background Th17 cells are critically involved in autoimmune disease induction and severity. Recently, we showed that Th17 cells, but not Th1 cells, from patients with rheumatoid arthritis (RA) upon interaction with RA-synovial-fibroblasts (RASF) directly induced autocrine IL-17A production. This autocrine IL-17A production in turn creates a pro-inflammatory loop characterised by up-regulation of the pro-inflammatory cytokines IL-6 and IL-8, and the cartilage degrading enzymes MMP-1 and MMP-3. This loop may be an important pathway in the progression of an early inflammatory arthritis, towards a chronic destructive arthritis. However, the mechanism underlying the autocrine IL-17A production in the proinflammatory loop upon Th17-RASF interaction is currently unknown.

Objectives To investigate the mechanism responsible for the autocrine IL-17A induction upon Th17-RASF interaction.

Materials and Methods CD4+CD45RO+CCR6+ (Th17) and CD4+CD45RO+CCR6- (Th1) cells were isolated by FACS sorting from healthy controls and early RA patients. These cells were cocultured with RASF, in the presence of neutralising antibodies directed against soluble IL-6R (anti-sIL-6R), and/or IL-1 $\beta$, and/or IL-23, etanercept, and celecoxib. Gene expression profiles were generated and supernatant was collected for cytokine analyses by ELISA.

Results IL-6, IL-1 $\beta$ and cyclooxygenase-2 (COX-2) expressions and prostaglandin- $\mathrm{E}_{2}\left(\mathrm{PGE}_{2}\right)$ production in Th17-RASF cultures were higher than in Th1-RASF cultures. Cytokine neutralisation showed that IL-1 $\beta$, IL- 6 and IL-23 contributed marginally to the IL-17A induction. In contrast, treatment with celecoxib, a COX-2 inhibiter, resulted in significantly lower $\mathrm{PGE}_{2}$ and IL-17A production. This effect was IL-17A specific as no inhibitory effects were found on IFN- $\gamma$ and TNF- $\alpha$ production. Combined celecoxib and TNF- $\alpha$ blockade more effectively suppressed the pro-inflammatory loop than did single treatment, as shown by lower IL-6, IL-8, matrix metalloproteinase-1 (MMP-1) and MMP-3 production.

Conclusions These findings show a critical role for the COX-2/ $\mathrm{PGE}_{2}$ pathway in driving Th17-mediated synovial inflammation in an IL-23 and monocyte independent manner. Therefore, it would be important to control $\mathrm{PGE}_{2}$ in chronic inflammation in RA and other Th17-mediated autoimmune disorders. 